\title{
The Front Line of Genomic Translation
}

\author{
Suzanne C. O'Neill, ${ }^{1}$ Colleen M. McBride, ${ }^{2}$ Angela D. Bryan, ${ }^{3}$ \\ Laura M. Koehly, ${ }^{2}$ and Louise Wideroff ${ }^{4}$ \\ ${ }^{1}$ Lombardi Comprehensive Cancer Center, Medical Center, Georgetown University, Washington, DC 20007, USA \\ ${ }^{2}$ Social and Behavioral Research Branch, National Human Genome Research Institute, NIH, MD 20892, USA \\ ${ }^{3}$ Department of Psychology, University of Colorado, CO 80309, USA \\ ${ }^{4}$ Division of Basic Neuroscience and Behavioral Research, National Institute on Drug Abuse, NIH, MD 20892, USA
}

Correspondence should be addressed to Suzanne C. O’Neill, sco4@georgetown.edu

Received 30 July 2012; Accepted 30 July 2012

Copyright ( 2012 Suzanne C. O’Neill et al. This is an open access article distributed under the Creative Commons Attribution License, which permits unrestricted use, distribution, and reproduction in any medium, provided the original work is properly cited.

Cancer prevention, detection, and treatment represent the frontline of genomic translation. Increasingly, new genomic knowledge is being used to inform personalized cancer prevention recommendations and treatment [1-3]. Genomic applications proposed and realized span the full cancer continuum, from cancer prevention and early detection vis a vis genomic risk profiles to motivate behavioral risk reduction and adherence [4] to screening and prophylactic prevention recommendations for high-risk families [5-7], to enhancing cancer survivorship by using genomic tumor profiles to inform treatment decisions and targeted cancer therapies $[8,9]$. Yet the utility for many of these applications is as yet unclear and will be influenced heavily by the public's, patients', and health care providers' responses and innumerous other factors, such as health care delivery models [3]. The contributors to this special issue consider various target groups' responses and contextual factors. To reflect the cancer continuum, the special issue is divided into three broad, overlapping themes - primary prevention, high risk families and family communication and clinical translation.

The issue begins with three papers that consider applications of genomic information to promote the primary prevention of cancer. Hay and colleagues present results of a randomized trial evaluating prototypic genetic risk feedback with first degree relatives of patients with melanoma. Using an experimental pre-post design, the authors tested whether varied feedback type (high risk mutation, gene-environment, nongenetic) and risk level (positive versus negative findings), all emphasizing the importance of family history regardless of feedback type, influenced families' risk perceptions, and behavioral intentions. Findings indicated that risk level, but not feedback type, was associated with higher perceived risk and behavioral intentions for sun protection and skin screening. It is widely acknowledged that efforts should be directed to building an evidence base to inform genomic risk communication practice. This will be essential as new technologies such as whole genome sequencing begin to yield voluminous amounts of risk information for providers, patients and their families. Thus unpacking the essential elements of effective risk communications could facilitate translation of emerging and increasingly complex genomic risk information.

Rutten and colleagues used data from the 2008 and 2011 waves of the Health Information National Trends Survey (HINTS), a nationally representative sample, to assess the public's awareness of direct to consumer (DTC) genetic testing. As expected, awareness of DTC genetic testing increased significantly (from $29.3 \%$ to $36.9 \%$ ) from 2008 to 2011. Awareness was higher in older adults who had more educational attainment, lived in urban settings, and who already have access to primary care. Awareness also was higher among those with a prior cancer diagnosis, $47.4 \%$ versus $35.9 \%$ of those without. Thus, affluent and highly educated individuals, as well as those affected by cancer, may be more aggressive information seekers about genetic tests. While DTC access to genetic testing is still highly controversial, these results suggest that such access does not have broad reach and thus may exacerbate existing health disparities.

Finally, in an effort to explore the underlying genetic contribution to exercise behavior, Karoly and colleagues explore associations between biologically plausible single nucleotide 
polymorphisms (SNPs) and response to exercise. Their results showed that SNPs in the FTO, CREB1, and OPRM1 genes as well as SNPs in SLIT2 and FAM5C were associated with physiological changes and subjective experiences during a session of moderate intensity exercise. Studies like this lay the groundwork for the eventual discovery of large panels of genetic markers that might help health promotion researchers to individually tailor behavioral interventions in ways that could optimize exercise adherence and ultimately decrease cancer incidence.

The second set of papers consider whether genomic risk assessments, including family health history assessments related to and genetic testing for hereditary cancer syndromes, have the potential to inform personalization of disease prevention and treatment behaviors. In order for this personalization to take place, patients must be proactive in informing their providers and their at-risk relatives about inherited disease risk, highlighting the importance of effective communication. Anderson and colleagues examine facilitators and barriers associated with young breast cancer survivors' use of genetics services, as early onset is an indicator of increased likelihood of carrying a mutation. One of the primary barriers to use genetic services was not having been referred to genetics education and risk evaluation by a cancer care provider. Vadaparampil and colleagues focused their attention on communication of test results among newly diagnosed women who were tested. While these survivors typically shared their BRCA1/2 results with their first-degree female relatives and their medical oncologists, communication was less likely to occur with male relatives or with physicians in other specialties, even their primary care providers. This again highlights multiple opportunities to foster communication within and outside the family. This need does not apply only to those affected with hereditary breast and ovarian cancer, as James and colleagues report that at least $20 \%$ of relatives at high risk of familial adenomatous polyposis (FAP) had not discussed their family history with a doctor or had a doctor explain their personal risk of cancer. Taken together, it is clear that reciprocal exchange of information, both within families and between families and their healthcare providers, is necessary for personalization of prevention recommendations and treatments to be realized. Thus, these papers point to the need for evaluating clinicfriendly approaches to expand reciprocal risk communication among patients, family members and providers.

The issue concludes with three papers that consider genomic tools in the oncology clinic. Specifically, within the past decade, gene-expression profiling and pharmacogenetic testing have become an integral part of clinical care for certain types of cancer. Oncotype DX, a 21-gene expression test performed on tumor tissue, has been used since 2004 to predict metastatic recurrence of early stage, estrogen receptor-positive breast cancer. Patients with elevated recurrence scores, indicative of more aggressive tumors, may opt for adjuvant chemotherapy as part of their treatment plan. In the immediate postmarketing period while awaiting further clinical trial results, some insurers considered the intermediate endpoint of clinical utility (i.e., whether testing has changed the course of treatment) to determine coverage [10]. While data have shown that patients with high recurrence scores tend to receive chemotherapy, the situation is not so clear for the $25 \%$ of tested patients with intermediate scores. In this special issue, Malo et al. report on a chart review of patients in an NCI-supported comprehensive cancer center who received Oncotype DX in 2004-2009. While the data should be interpreted in the context of a small, single site study, after adjustment for a clinical and sociodemographic variables, both high and intermediate recurrence score remained significantly associated with chemotherapy uptake. In turn, Sulayman and colleagues address the psychosocial aspects of Oncotype DX testing, focusing on the joint effects of recurrence score and treatment decision style. They find that passive decision style was associated with higher distress and lower quality of life, particularly among patients with intermediate recurrence scores. Past patient-reported outcomes research on predictive genetic tests for $B R C A 1 / 2$ mutations have enabled genetic counselors and physicians to better integrate counseling into care, thus these results too could result in improved quality of life for carriers and family members [11]. The issue concludes with Cox et al.'s presentation of data from a survey of Oregon health providers' use and knowledge of genebased predictive and diagnostic tests, as well as pharmacogenetic tests for drug response. Not surprisingly, clinicians reported greater familiarity with tests for which practice guidelines are available, such as tests for breast/ovarian and Lynch syndromes. Although knowledge and test use differed by specialty, many reported limited knowledge of medical genetics and gave lack of familiarity with particular tests as the main reason for not using them in practice. This lag time between commercialization of various genetic tests and widespread adoption in practice presents challenges for effective translation. Accelerated clinical research is needed to inform new practice guidelines as are systematic efforts to build clinicians' competencies in this area.

The articles in this special issue represent a wide range of populations, settings, and contexts.

\section{Acknowledgment}

We thank the contributing authors and the anonymous reviewers who made this special issue possible. We hope that this issue will stimulate more research at the frontline of translational cancer genomics, behavioral science, clinical care, and public health.

Suzanne C. O'Neill
Colleen M. McBride
Angela D. Bryan
Laura M. Koehly
Louise Wideroff

\section{References}

[1] J. N. Weitzel, K. R. Blazer, D. J. MacDonald et al., "Genetics, genomics and cacner risk assessment: state of the art and future directions in the era of personalized medicine," $A$ Cancer Journal for Clinicians, vol. 61, no. 5, pp. 327-359, 2011. 
[2] U. McDermott, J. R. Downing, and M. R. Stratton, "Genomics and the continuum of cancer care," The New England Journal of Medicine, vol. 364, no. 4, pp. 340-350, 2011.

[3] M. J. Khoury, R. J. Coates, and M. L. Fennell, "Multilevel research and the challenges of implementing genomic medicine.," Journal of the National Cancer Institute Monograph, vol. 2012, no. 44, pp. 110-120, 2012.

[4] C. M. McBride, A. D. Bryan, M. S. Bray et al., "Health behavior change: can genomics improve behavioral adherence?" American Journal of Public Health, vol. 102, no. 3, pp. 401-405, 2012.

[5] K. A. Metcalfe, N. Mian, and M. Enmore, "Long-term followup of Jewish women with a BRCA1 and BRCA2 mutation who underwent population genetic screening," Breast Cancer Research and Treatment, vol. 1332, pp. 735-740, 2012.

[6] S. M. Domchek, T. M. Friebel, C. F. Singer et al., "Association of risk-reducing surgery in BRCA1 or BRCA2 mutation carriers with cancer risk and mortality," Journal of the American Medical Association, vol. 304, no. 9, pp. 967-975, 2010.

[7] M. D. Schwartz, C. Isaacs, K. D. Graves et al., "Long-term outcomes of BRCA1/BRCA2 testing: risk reduction and surveillance," Cancer, vol. 118, no. 2, pp. 510-517, 2012.

[8] S. Paik, G. Tang, S. Shak et al., "Gene expression and benefit of chemotherapy in women with node-negative, estrogen receptor-positive breast cancer," Journal of Clinical Oncology, vol. 24, no. 23, pp. 3726-3734, 2006.

[9] L. Harris, H. Fritsche, R. Mennel et al., "American society of clinical oncology 2007 update of recommendations for the use of tumor markers in breast cancer," Journal of Clinical Oncology, vol. 25, no. 33, pp. 5287-5312, 2007.

[10] J. R. Trosman, S. L. Van Bebber, and K. A. Phillips, "Coverage policy development for personalized medicine: private payer perspectives on developing policy for the 21-gene assay," Journal of Oncology Practice, vol. 6, no. 5, pp. 238-242, 2010.

[11] D. Braithwaite, J. Emery, F. Walter, A. T. Prevost, and S. Sutton, "Psychological impact of genetic counseling for familial cancer: a systematic review and meta-analysis," Familial Cancer, vol. 5, no. 1, pp. 61-75, 2006. 


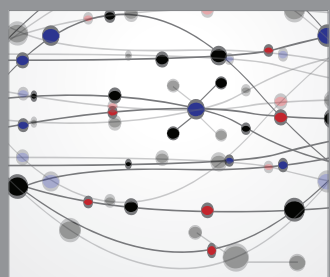

The Scientific World Journal
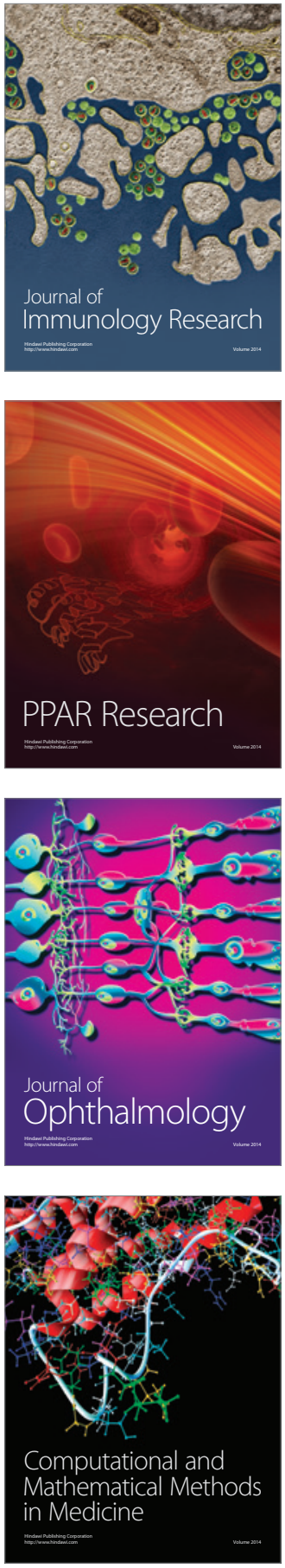

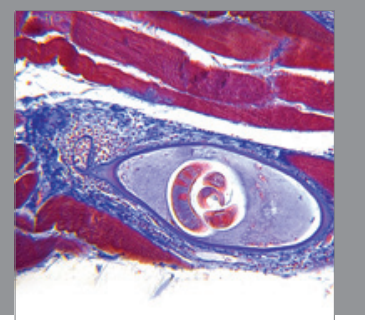

Gastroenterology

Research and Practice
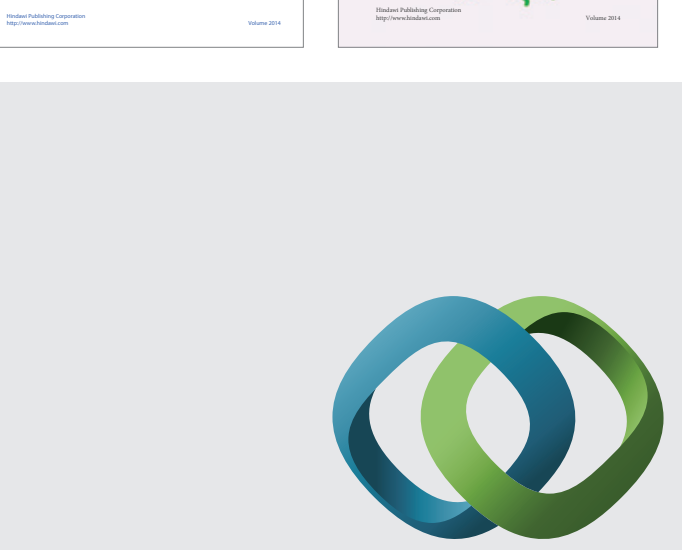

\section{Hindawi}

Submit your manuscripts at

http://www.hindawi.com
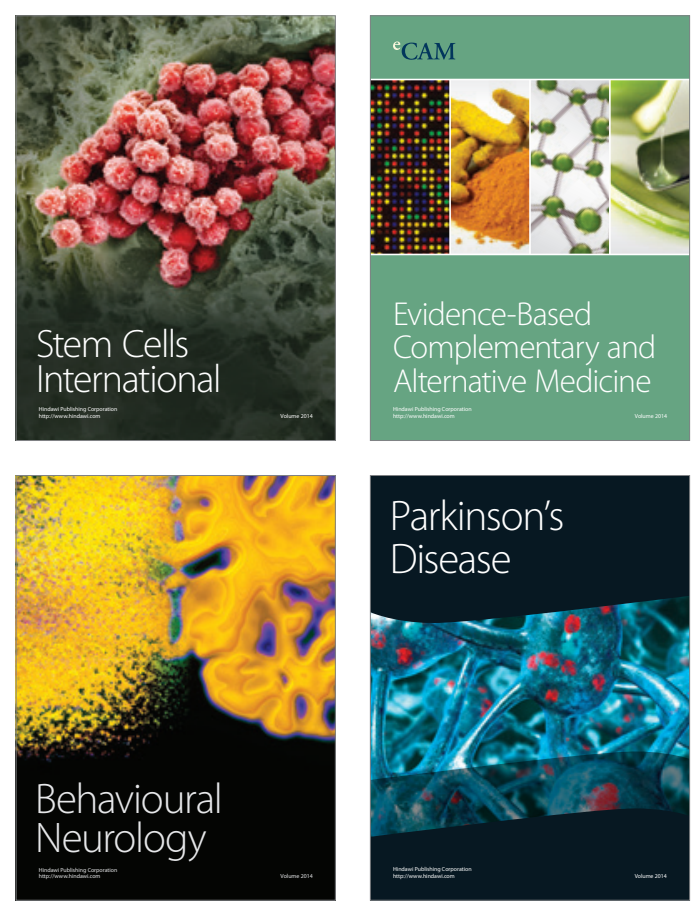

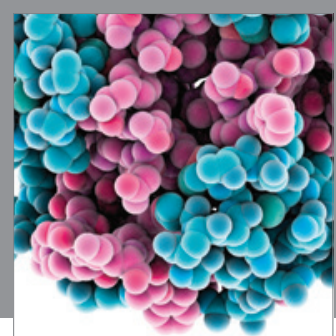

Journal of
Diabetes Research

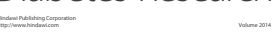

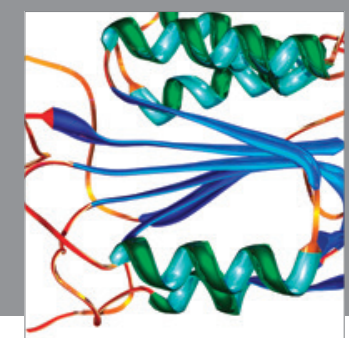

Disease Markers
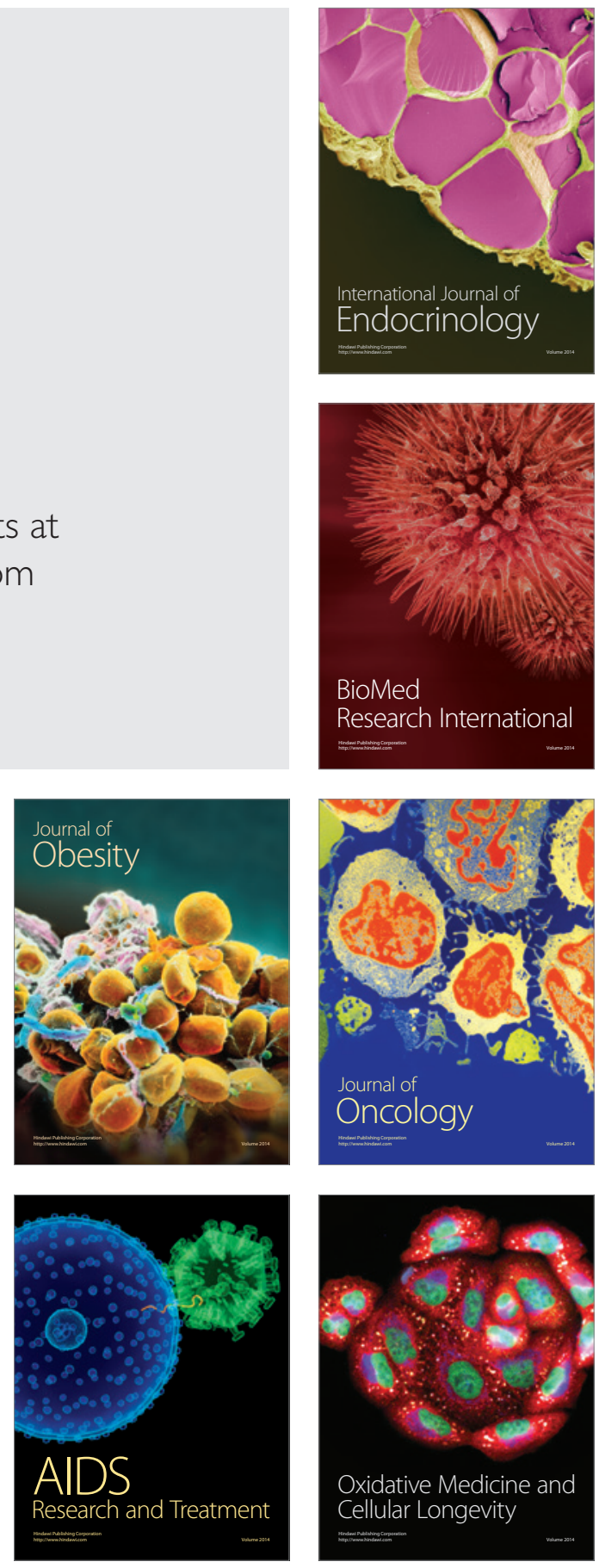